\title{
On the behavior of lightning induced voltage
}

\begin{abstract}
In this paper, the influence of lightning induced voltages (LIV) on distribution lines (DL) is considered and the effects of channel and also geometrical parameters on the values of LIV are processed and the results are discussed accordingly. The results show that the peak values LIV are more dependent on the current peak, return stroke velocity and the conductor height with a linear relationship. Therefore, in order to design or improve distribution networks, the behaviour of LIV under different channel and geometrical conditions can be useful for setting and optimizing the appropriate protection level on the lines.
\end{abstract}

Keyword: Lightning induced voltage; Lightning; Return stroke 